\title{
CD27-lgD- memory B cells are modulated by in vivo interleukin-6 receptor (IL-6R) blockade in rheumatoid arthritis
}

\author{
Zafar Mahmood ${ }^{1 *}$, Khalid Muhammad ${ }^{1,2}$, Marc Schmalzing $^{1}$, Petra Roll ${ }^{1}$, Thomas Dörner ${ }^{3}$ and Hans-Peter Tony ${ }^{1 *}$
}

\begin{abstract}
Introduction: Enhanced B cell activity, particularly memory B cells have gained interest in evaluating response during therapies with biologics. CD27-lgD- double-negative (DN) B cells lacking the conventional memory marker CD27 are reported to be part of the memory compartment, however, only scarce data is available for rheumatoid arthritis (RA). We therefore focused on DN B cells in RA, studied their isotypes and modulation during interleukin-6 receptor (IL-6R) inhibition by tocilizumab (TCZ).

Methods: DN B cells were phenotypically analyzed from 40 RA patients during TCZ at baseline week 12, week 24 and 1 year. A single $B$ cell polymerase chain reaction (PCR) approach was used to study lg receptors, $V_{H}$ gene rearrangements and specific isotypes.

Results: Phenotypic analysis showed a significantly expanded population of DN B cells in RA which contain a heterogeneous mixture of lgG-, IgA- and IgM-expressing cells with a clear dominance of IgG+ cells. DN B cells carry rearranged heavy chain gene sequences with a diversified mutational pattern consistent with memory $B$ cells. In contrast to tumor necrosis factor alpha (TNF-a) inhibition, a significant reduction in mutational frequency of BCR gene rearrangements at week 12, 24 and 1 year $(P<0.0001)$ was observed by in vivo IL-6R inhibition. These changes were observed for all BCR isotypes IgG, IgA and IgM at week 12, 24 and 1 year $(P<0.0001)$. IgA-RF, IgA serum level and IgA+ DN B cells decreased significantly $(P<0.05)$ at week 12 and week 24 during TCZ. Patients with a good European League Against Rheumatism (EULAR) response to TCZ had less DN B cells at baseline as compared to moderate responders $(P=0.006)$. Univariate logistic regression analysis revealed that the frequency of DN B cells at baseline is inversely correlated to a subsequent good EULAR response $(P=0.024)$ with an odds ratio of $1.48(95 \%$ confidence interval as 1.05 to 2.06).
\end{abstract}

Conclusions: In RA, the heterogeneous DN B cell compartment is expanded and dominated by lgG isotype. TCZ can modulate the mutational status of DN Ig isotype receptors over 1 year. Interestingly, the frequency of DN B cells in RA may serve as a baseline predictor of subsequent EULAR response to TCZ.

\section{Introduction}

Rheumatoid arthritis (RA) is a chronic, systemic, inflammatory, autoimmune disease characterized by inflammation of the joints, which results in their progressive destruction [1,2]. Recent studies suggest that B cells play an important role in the development and progression of RA through several mechanistic pathways, and the subsequent polyclonal activation of B cells [3]. By producing

\footnotetext{
*Correspondence: Mahmood_Z@ukw.de; Tony_H@ukw.de

'Department of Medicine II, Rheumatology and Clinical Immunology,

University of Würzburg, Oberdürrbacher Str. 6, D-97080 Würzburg, Germany Full list of author information is available at the end of the article
}

different inflammatory cytokines and autoantibodies, they may directly drive pathologic inflammation [4]. Moreover, memory B cells have gained particular interest in evaluating response during therapies from biologics that have shown promising results in treatment of RA. Different cytokines are involved in B cell differentiation, maintenance and survival. They are distinctly implicated in each phase of the pathogenesis of RA-promoting autoimmunity, maintaining chronic inflammatory synovitis and driving the destruction of joint tissue [5]. Biological agents targeting key proinflammatory cytokines, such as tumor necrosis factor alpha (TNF- $\alpha$ ) and interleukin 6 (IL-6) 
have been substantially advanced in the treatment of autoimmunity [6]. IL-6 is a multifunctional pleiotropic cytokine acting as stimulator of both $\mathrm{B}$ and $\mathrm{T}$ cell functions. It is produced by various cells of the innate immune system (for example macrophages, dendritic cells, mast cells, neutrophils), B cells, and to some extent by CD4 effector T helper (Th) cells [7]. IL-6 also influences various cell types and has multiple biological activities through its unique receptor system [8]. It has been described as a late-acting $B$ cell differentiation factor that is involved in in vitro differentiation of B cells into antibody-forming cells and germinal center reactions. In addition to its involvement in immune responses, it also regulates hematopoiesis, the acute phase response and inflammation. Dysregulation of IL-6 production and its pathological role in different autoimmune diseases have been well documented and highlight IL- 6 and its signaling cascade as a potential target for autoimmune therapy [9-13]. Consequently, tocilizumab (TCZ), a humanized anti-IL-6 receptor (IL-6R) monoclonal antibody (mAb) against the alpha chain of IL-6R, which prevents binding of IL-6 to membrane and soluble IL-6R, was developed and has been licensed for the treatment of RA [14]. TCZ has shown convincing clinical efficacy by reduction of signs/symptoms and a marked inhibition of radiological progression [11].

Functionally distinct B cell subsets can be defined by the phenotype expression of CD27 and immunoglobulin D (IgD). Human peripheral memory B cells are mainly discriminated from naïve $\mathrm{B}$ cells by the phenotypic expression of CD27 (a member of the tumor necrosis factor receptor (TNFR) family) and presence of somatic hypermutation (SHM) in their Ig variable genes $[15,16]$. CD27 expression by B cells has been considered a hallmark for SHM and their memory. CD27+ memory B cells are a heterogeneous population comprising of pre-switch $(\mathrm{IgD}+\mathrm{CD} 27+)$ and post-switch (IgD-CD27+) B cell subsets $[13,17,18]$. There are still unanswered questions about the exact identification of memory B cells based on CD27 expression, since recent studies in these lines have shown a double-negative (DN) population (CD19+CD27-IgD-) that bears all signatures of memory $B$ cells [19-21] (Figure 1A). A very large portion of DN (CD27-IgD-) B cells express mutated Ig and an evaluation of telomere

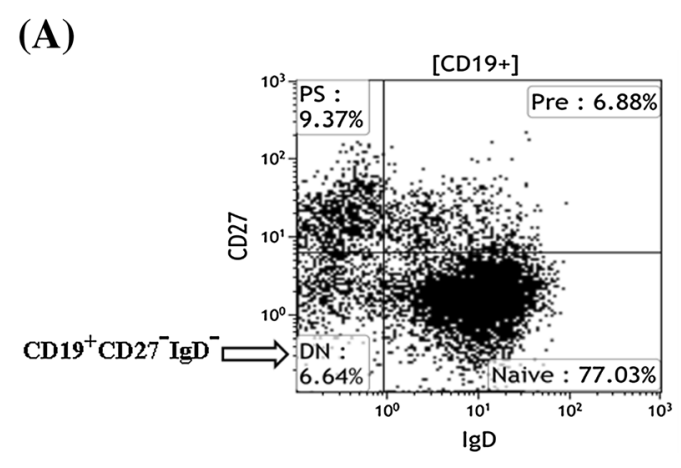

(A)

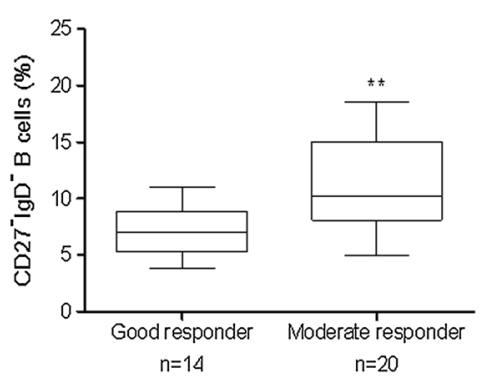

(B)

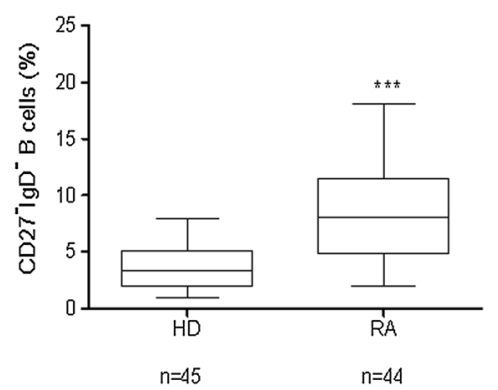

(D)

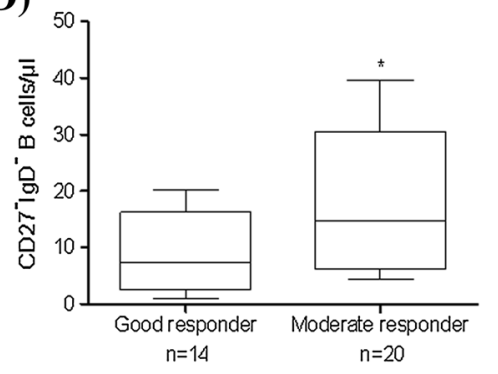

Figure 1 Phenotype analysis of CD27-IgD- B cells in RA patients and their relation to EULAR response. (A) Representative FACS plot. Characterization of (CD27-lgD-) DN B cells, PS = post-switch (CD27 + lgD-), Pre = pre-switch (CD27 + lgD+) and naïve (CD27-lgD+) B cells. (B) Comparison of DN B cells in RA patients and HD. DN B cells in RA patients $(n=44)$ and $H D(n=45)$ show a significantly higher percentage of the frequency of DN B cells in RA patients $(P<0.0001)$. (C) EULAR response to IL-6R inhibition. Week 12 EULAR good responders (BL DAS28 $=$ $5.1 \pm 0.3)$ to TCZ have significantly $(P=0.006)$ lower frequency of DN B cells at baseline compared to EULAR moderate responders (BL DAS28 = $5.3 \pm 0.3)$. (D) EULAR responses to IL-6R inhibition (absolute cell numbers). Week 12 EULAR good responders have significantly $(P=0.05)$ lower absolute DN B cell numbers at baseline compared to moderate responders. Data shown in box-whisker plot where boxes represent 25th to 75 th percentiles and the lines within the boxes represent the median. $P$ values were determined by Mann-Whitney $U$ test using GraphPad Prism 5. $\left({ }^{* * *} P<0.0001,{ }^{* *} P<0.001\right.$ and $\left.{ }^{*} P<0.05\right)$. BL DAS28, baseline disease activity score 28; DN, double-negative; EULAR, European League Against Rheumatism; HD, healthy donor; Ig, immunoglobulin; IL-6R, interleukin-6 receptor; RA, rheumatoid arthritis; TCZ, tocilizumab. 
length, expression of the anti-apoptotic molecule $\mathrm{Bcl} 2$, and absence of the ATP-binding cassette B1 transporter (ABCB1) have been used to discriminate them from naïve $C D 27-B$ cells and relate them to the memory $B$ cell compartment [22,23]. Even though DN memory B cells mainly express switched Ig isotypes, they have a reduced rate of SHM compared to post-switch B cells. This has been hypothesized to be due to either an impaired germinal center (GC) formation or resembling a distinct lineage of memory B cells [23,24]. In systemic lupus erythematosus (SLE), DN B cells are expanded and could be linked to autoimmunity by analysis of the specific autoantibodies including 9G4 expression [19]. So far, the nature of DN B cells has still not been fully delineated in general as well as in autoimmune diseases.

Our previous studies of memory B cell subsets during in vivo IL-6R inhibition indicated phenotypic and molecular changes in pre-and post-switch memory B cells $[13,14,25]$. In RA, DN B cells have not been thoroughly studied and there is scarce information in the literature. Therefore, we initiated the current study to analyze the DN B cell compartment in RA in more detail by phenotypic and molecular analyses of the different isotypic DN $B$ cell receptors, their immunoglobulin receptor (Ig-R) mutational pattern and their modulation by in vivo IL$6 \mathrm{R}$ and TNF- $\alpha$ inhibition.

\section{Methods}

\section{Patients and healthy donors}

Peripheral blood was taken from 44 rheumatoid arthritis patients (RA) with a median age of 54 (range 33 to 79) years and 49 healthy age-matched donors (HD) for B cell phenotype and molecular analysis. All patients met the American College of Rheumatology revised criteria for RA [26]. The patients had median disease duration of 9 (range 2 to 33) years and 77\% were female. Informed consent was obtained from all patients according to the protocol approved by the ethics committee of the university hospital, Würzburg, Germany. Patients who failed to respond to treatment with standard diseasemodifying antirheumatic drugs (DMARDs) including methotrexate (MTX) were eligible and enrolled in the study. A dose of $8 \mathrm{mg} / \mathrm{kg}$ TCZ was administered every 4 weeks as a 60-minute infusion in combination with MTX. Alternatively, adalimumab (ADA) (40 mg every 2 weeks) in combination with MTX was given as control. The clinical primary end point was set at 12 weeks, with an extension period up to 24 weeks and follow-up till 1 year. Forty TCZ-treated patients were followed at baseline, weeks 12 and 24 for phenotypic analysis. A total of $33 / 40$ patients were rheumatoid factor (RF)-positive and 28/40 patients were anti-citrullinated protein antibodies (ACPA)-positive. Furthermore, all the ACPA-positive patients were positive for RF, 5/40 ACPA-negative patients were RF-positive and 7/40 patients were negative for both RF and ACPA. Four patients left the study after week 12 . For correlation with clinical parameters, 36 patients undergoing TCZ therapy were studied, 14/36 reached a European League Against Rheumatism (EULAR) good response at week 12, 20/36 were EULAR moderate responders and 2/36 were non-responders to TCZ. The baseline disease activity score using 28 joint counts (DAS28) and C-reactive protein (CRP) levels of EULAR good responders $(5.1 \pm 0.3 ; 0.9 \pm 0.2 \mathrm{mg} / \mathrm{dl})$ and moderate responders $(5.3 \pm 0.3 ; 0.8 \pm 0.2 \mathrm{mg} / \mathrm{dl})$, respectively were similar [27]. Clinical characteristics of the patients undergoing TCZ therapy are summarized in Table 1. Nine RA patients under TCZ therapy, four patients under anti-TNF- $\alpha$ therapy and three HD were selected for analysis of mutational patterns of Ig-Rs by single-cell polymerase chain reaction (PCR) technique.

\section{Flow cytometric analysis}

Whole blood staining was used for phenotype studies and peripheral blood mononuclear cells (PBMCs) were used for single-cell PCR approach. Whole blood and PBMCs were stained with the following monoclonal antibodies: antiCD45-krome orange (Beckman Coulter (Brea, CA, USA), cat no. 96416), anti-CD14-PC5.5 (Beckman Coulter, cat no. A70204), anti-CD19-APC-Alexa Fluor 750 (Beckman Coulter, cat no. A94681), anti-CD19-APC (BD Pharmingen (San Jose, CA, USA), cat no. 555415), anti-CD27-PE (BD Pharmingen, cat no. 555441), anti-CD27-PC7 (Beckman Coulter, cat no. A54823), anti-CD27-ECD (Beckman Coulter, customized), anti-IgD-FITC (BD Pharmingen, cat no. 555778), anti-IgA-FITC (Beckman Coulter, cat no. 732610), anti-IgG-PECy7 (BD Pharmingen, cat no. 561298) and anti-IgM-APC (BioLegend (San Diego, CA, USA) cat no. 314510). After staining, cells were analyzed by 10 -color flow cytometer (Navios, Beckman Coulter). B cells were identified by CD19+ cells gated on CD45 + CD14- lymphocytes in combination with forward scatter versus side scatter gating on CD45+ lymphocytes. B cell subpopulations were identified by using CD27 and IgD expression gated on B cells. Expression of IgG, IgA and IgM was analyzed for CD27 + IgD- post-switch and CD27-IgD- DN B cells, respectively. At least 20,000 $\mathrm{CD} 19+$ events were collected for each analysis. The total number of B cells of various phenotypes was calculated per microliter of blood, based on the frequency of these cells among the lymphocytes and the absolute number of white blood cells.

\section{Single-cell sorting}

PBMCs were isolated by Ficoll-Paque Plus separation (Pharmacia Biotech, Freiburg, Germany) using the standard protocol. Single B cell sorting from PBMCs was carried out as described previously [28]. Briefly, B cells were 
Table 1 Characteristics of patients treated with tocilizumab therapy

\begin{tabular}{|c|c|c|c|c|}
\hline & Baseline $(n=36)$ & Week $12(n=36)$ & Week $24(n=36)$ & 1 year $(n=36)$ \\
\hline Age, median (range) years & $54(33-79)$ & & & \\
\hline$\%$ female & $77 \%$ & & & \\
\hline Disease duration, median (range) years & $9(2-33)$ & & & \\
\hline RF positive & 36 & & & \\
\hline ACPA positive & 28 & & & \\
\hline \multirow[t]{2}{*}{ RF/ACPA positive } & 28 & & & \\
\hline & mean \pm SEM & & & \\
\hline DAS28 score & $5.2 \pm 0.3$ & $3.3 \pm 0.3^{\psi}$ & $2.7 \pm 0.2^{\psi}$ & $1.9 \pm 0.2^{\psi}$ \\
\hline CRP, mg/dl & $0.9 \pm 0.1$ & $0.14 \pm 0.06^{\psi}$ & $0.09 \pm 0.03^{\psi}$ & $0.06 \pm 0.02^{\psi}$ \\
\hline ESR, mm/hour & $32.1 \pm 3.4$ & $9.8 \pm 1.6^{\psi}$ & $7.2 \pm 0.9^{\psi}$ & $5.6 \pm 0.8^{\psi}$ \\
\hline Patient's VAS & $58.3 \pm 4.4$ & $48.6 \pm 4.8^{\psi}$ & $34.8 \pm 5.4^{\Psi}$ & $21.5 \pm 4.8^{\psi}$ \\
\hline IgA-RF U/ml & $122.3 \pm 33.3$ & $99.9 \pm 26.3^{\psi}$ & $63.3 \pm 19.8^{\psi}$ & - \\
\hline IgA total $\mathrm{U} / \mathrm{ml}$ & $265.4 \pm 20.8$ & $237.2 \pm 19.5^{\psi}$ & $203.1 \pm 18.9^{\psi}$ & - \\
\hline
\end{tabular}

The primary end point of the study was a reduction in the disease activity score in 28 joints (DAS28) at week 12. Except where indicated otherwise, values are the mean \pm standard error of the mean (SEM). ${ }^{\Psi} P<0.05$ versus baseline and Mann-Whitney $t$ test was used for statistics. RF, rheumatoid factor; ACPA, anti-citrullinated protein antibodies; CRP, C-reactive protein; ESR, erythrocyte sedimentation rate; VAS, visual analog scale (100 mm); IgA, immunoglobulin A.

stained for CD19-APC, CD27-PE and IgD-FITC and gated by forward/side scatter; doublets for CD19 positivity were excluded. DN B cells were defined as CD19 + IgD-CD27-. Individual DN B cells were sorted in a 96-well plate containing lysis buffer by using FACS ARIA-III cell sorter (Becton Dickinson, San Jose, CA, USA). Lysis buffer was comprised of Triton X-100, bovine serum albumin (BSA), oligo $(\mathrm{dT})_{15}$ primer, dithiothreitol, RNasin, and RNAsefree double-distilled $\mathrm{H}_{2} \mathrm{O}$ or RNAse-free $\mathrm{H}_{2} \mathrm{O}$.

\section{CDNA preparation and nested PCR}

A mixture comprising RT-PCR buffer, reverse transcriptase and nucleotides from Titan One Tube RT-PCR System (Roche Diagnostics, Mannheim, Germany) was added into a 96-well plate. cDNA synthesis was carried out at $50^{\circ} \mathrm{C}$ for 1 hour. $\mathrm{V}_{\mathrm{H}} 3$ gene rearrangements comprising the largest $\mathrm{V}_{\mathrm{H}}$ family were amplified by nested PCRs using family-specific primers as previously described [29]. Briefly, the cycle program consisted of one cycle at $95^{\circ} \mathrm{C}$ for $5 \mathrm{~min}, 50$ to $58^{\circ} \mathrm{C}$ for $1 \mathrm{~min}, 72^{\circ} \mathrm{C}$ for $1 \mathrm{~min}$, followed by 30 cycles of $94^{\circ} \mathrm{C}$ for $1 \mathrm{~min}, 50$ to $58^{\circ} \mathrm{C}$ for $30 \mathrm{~s}, 72^{\circ} \mathrm{C}$ for $1 \mathrm{~min}$, followed by $5 \mathrm{~min}$ incubation at $72^{\circ} \mathrm{C}$. Using $5 \mu \mathrm{l}$ of the PCR product from first amplification as template, the second round of nested PCR was conducted with a primer specific. In order to differentiate different isotypes in DN B cells, $\mathrm{C}_{\mathrm{H}} 1 \mu-, \mathrm{C}_{\mathrm{H}} 1 \alpha-$ and $\mathrm{C}_{\mathrm{H}} 1 \gamma$-specific PCRs were carried out using cycle program as described previously [30]. The error rate due to the Taq polymerase used in the amplification process was estimated to be $1 \times 10^{-4}$ mutations/bp [31]. $\mathrm{V}_{\mathrm{H}}$ familyspecific PCR products (350 bp) were separated via electrophoresis using $1.5 \%$ agarose gel and further purified by using MinElute Gel Extraction kit (Qiagen, Hilden, Germany) according to the manufacturer's instructions.

\section{Sequencing and analysis}

Gel extracted PCR products of $\mathrm{Ig}_{\mathrm{H}} \mathrm{V} 3$ and isotypespecific Ig were amplified using BigDye Terminator Cycle Sequencing Ready Reaction kit followed by sequencing in genetic analyzer ABI PRISM 310 (Applied Biosystems, Carlsbad, CA, USA). A total of 2,407 sequences were analyzed by matching their closest germline counterparts using the online program JOINSOLVER [32].

\section{Statistical analysis}

Statistical analysis was performed using GraphPad Prism 5.0 (GraphPad Software, San Diego, CA, USA) and SPSS Statistics 22.0 (IBM Corp., Armonk, NY, USA). Values were always compared with baseline levels by using the nonparametric Wilcoxon matched pair test and MannWhitney $U$ test. Univariate logistic regression was used to calculate odd ratios and correlation using Pearson $r$. The values $\leq 0.05$ were considered to be significant. ${ }^{* * * * *} P<0.0001$, ${ }^{* * *} P<0.001$ and $" P<0.01$.

\section{Results}

Double-negative (CD19 + IgD-CD27-) B cells are expanded in RA

Based on surface expression of IgD and CD27 (Figure 1A), human peripheral CD19+B cells were divided into four subsets: mature naïve $\mathrm{B}$ cells (IgD + CD27-), pre-switch memory B cells $(\mathrm{IgD}+\mathrm{CD} 27+)$, post-switch memory B cells (IgD-CD27+) and DN B cells (IgD-CD27-). Frequencies of peripheral DN B cells were determined in RA 
patients $(\mathrm{n}=44$; median age approximately 54 years) and healthy donors $(n=45$; median age approximately 52 years). We found significantly enhanced frequencies of DN B cells in RA patients compared to HD (Figure 1B). Here, RA patients had a median (range) of 8.4 (2.0 to 18.1) percent of CD19+ DN B cells as compared with 3.3 (1.0 to 7.9) percent in HD. Notably, absolute numbers of DN B cells of RA with 10.7 (1.9 to 32.9 ) cells/ $\mu$ l were comparable to HD with 9.6 (1.7 to 29.5$)$ cells/ $\mu \mathrm{l}$.

\section{DN B cells correlate to clinical response to tocilizumab}

During TCZ therapy, DAS28 of all patients declined significantly from 5.2 at baseline (BL) to 3.3, 2.7 and 1.9 at week 12,24 and 1 year $(P<0.0001)$. The inflammatory parameters erythrocyte sedimentation rate (ESR) and CRP were also significantly reduced (Table 1 ). The clinical response was maintained up to 1 year and there were no serious adverse events or infections during the study. At week 12, during IL-6 inhibition with TCZ therapy 14 out of 36 patients reached a EULAR good response, 20 patients reached a moderate response and 2 patients were non-responders. Interestingly, we found that good EULAR responders to TCZ at week 12 had a significantly lower frequency of $\mathrm{DN} B$ cells compared to moderate responders, at BL. The same correlation was also found for absolute numbers of DN B cells at BL (Figure 1C-D). There was no significant correlation between frequency of DN B cells with DAS28 $(\mathrm{R}=-0.007 ; P=0.97)$ and CRP levels $(\mathrm{R}=0.07 ; P=0.70)$. Univariate logistic regression analysis revealed that the frequency of DN B cells at BL is inversely correlated with a subsequent EULAR good response with a significant $(P=0.024)$ odds ratio of 1.48 (95\% confidence interval $(\mathrm{CI})$ as 1.05 to 2.06 ) favoring EULAR good response. This indicates that the percentage of DN B cells is elevated in RA and correlated with EULAR response to TCZ.

\section{Phenotypic isotype distribution of CD27-IgD- DN and post-switch $B$ cells in RA and their modulation during IL-6R inhibition}

In order to reveal the isotypic distribution of DN B cells, we measured the surface expression of IgG, IgA and IgM isotypes and followed them in RA patients $(n=36)$ during TCZ treatment till week 24. DN B cells showed a heterogeneous mixture of cells containing IgG+, IgA+ and IgM+ expressing B cells with a dominance of IgG isotype. In detail, DN B cells contained a median (range) of $60.6 \%$ (40.9 to 86.7 ) IgG+ isotype of total DN B cells, $24.7 \%$ (10.0 to 64.2 ) of IgA+, and $8.0 \%$ (2.3 to 25.8 ) of IgM+ B cells. For comparison, post-switch memory B cells displayed an almost equal distribution of IgG+ and IgA+ cells; $45.3 \%$ (26.2 to 67.3 ) IgG+ and $42.7 \%$ (24.5 to 57.4) IgA+ (Figure 2). The distribution of IgG+, IgA+ and IgM+ DN B cells in HD were comparable to RA patients (Additional file 1). Since IL-6 inhibition has been shown to influence $B$ cell maturation, we studied DN B cells during TCZ therapy. TCZ did not change the elevated frequency of the overall population of CD27IgD- DN B cells. However, by analyzing the Ig isotypes in CD27-IgD- DN B cells we found a significant relative decrease of IgA+ DN cells from $24.7 \%$ (10.0 to 64.2) median (range) to $18.4 \%$ (4.8 to 34.7$)$ at week $12(P=0.004)$ and $20.5 \%$ ( 4.6 to 33.8$)$ at week $24(P=0.04)$, respectively. We did not find any remarkable changes in IgG+ or IgM+ B cells (Figure 2B). Moreover IgA+ DN B cells declined also significantly in absolute numbers $(P<0.05)$ Additional file 2A). In contrast, the frequency of both IgA+ and IgG+ in the post-switch B cells compartment was not influenced during TCZ therapy (Figure $2 \mathrm{C}$ ), indicating a more dynamic IgA response in the DN compartment. Interestingly serum IgA levels as well as RF-IgA both declined significantly during TCZ therapy (Table 1).

\section{Somatically mutated Ig-receptors of DN B cells and their modulation during IL-6R}

In a subsequent study we performed molecular analysis of DN B cells and analyzed in detail the Ig-R of individually sorted cells from HDs $(\mathrm{n}=3)$ and from RA patients during TCZ $(n=9)$ or anti-TNF- $\alpha$ therapy $(n=4)$. As shown previously, [33] $I g-\mathrm{V}_{\mathrm{H}} 3$ gene rearrangements of $\mathrm{DN}$ B cells Ig-R showed less mutation compared to pre-switch and post-switch B cells. Specifically, mutational frequency of DN B cells was $4.0 \pm 0.2 \%$ compared to $4.5 \pm 0.2 \%$ for pre-switch B cells and $6.2 \pm 0.3 \%$ for post-switch B cells. Moreover, the mutational frequency of DN B cells was comparable in RA and HD (Additional file 2A).

During TCZ therapy however, the mutational frequency of Ig-R of DN B cells was significantly reduced at week 12, week 24 and 1 year (Figure 3A). The mutational frequency of DN B cells decreased from 4.04 \pm $0.2 \%$ (BL) to $2.52 \pm 0.2 \%$ at week $12(P<0.0001), 1.98 \pm$ $0.3 \%$ at week $24(P<0.0001)$ and $1.89 \pm 0.3 \%(P<0.0001)$ at 1 year respectively during TCZ (Figure $3 \mathrm{~A}$ ). Consistent with that, highly mutated sequences ( $>20$ mutations per sequence) decreased from $46.2 \pm 5.1 \%(\mathrm{BL})$ to $28.7 \pm$ $5.5 \%$ at week $12(P=0.018), 23.1 \pm 6.8 \%$ at week $24(P=$ $0.035)$ and $17.3 \pm 3.9 \%$ at 1 year $(P=0.0003)$ during TCZ therapy. In parallel, a significant increase of unmutated (0 mutation per sequence) sequences from $15.1 \pm 3.4 \%$ (BL) to $22.7 \pm 3.7 \%$ at week $12(P=0.05), 32.1 \pm 4.6 \%$ at week $24(P=0.005)$ and $37.1 \pm 4.6 \%$ at 1 year $(P=0.004)$ was found (data not shown). In order to evaluate TCZspecific influences on Ig gene rearrangements, we also studied patients $(n=4)$ who underwent TNF- $\alpha$ inhibition using adalimumab therapy (Figure 3B). Mutational frequency of Ig-R did not change in DN B cells during adalimumab therapy (Figure 3B). Both therapeutic anticytokine interventions significantly reduced clinical 


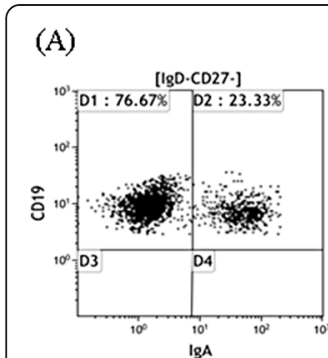

(B)

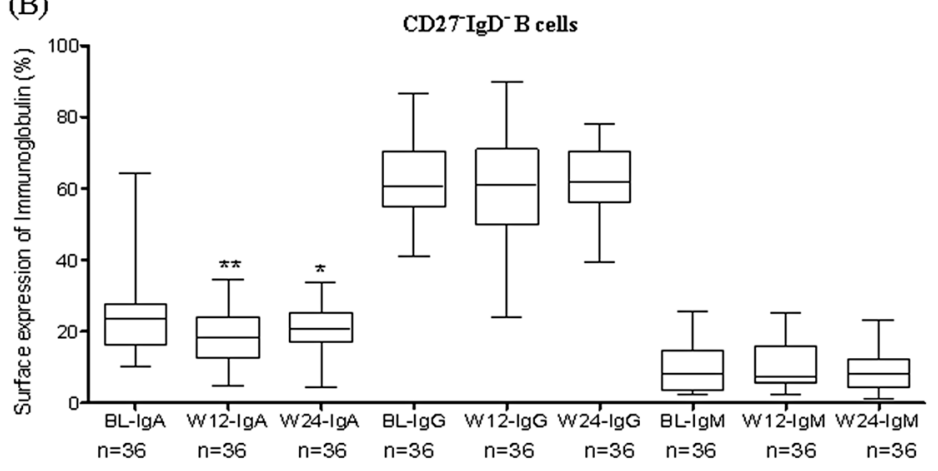

CD27 IgD ${ }^{-B}$ cells
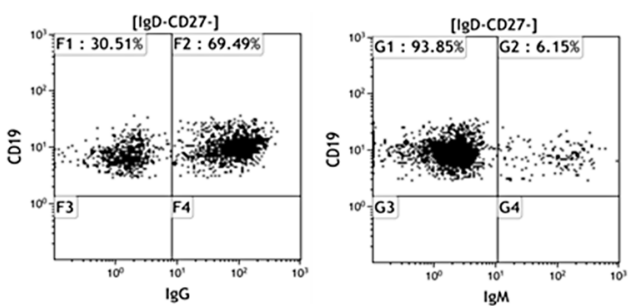

(g)
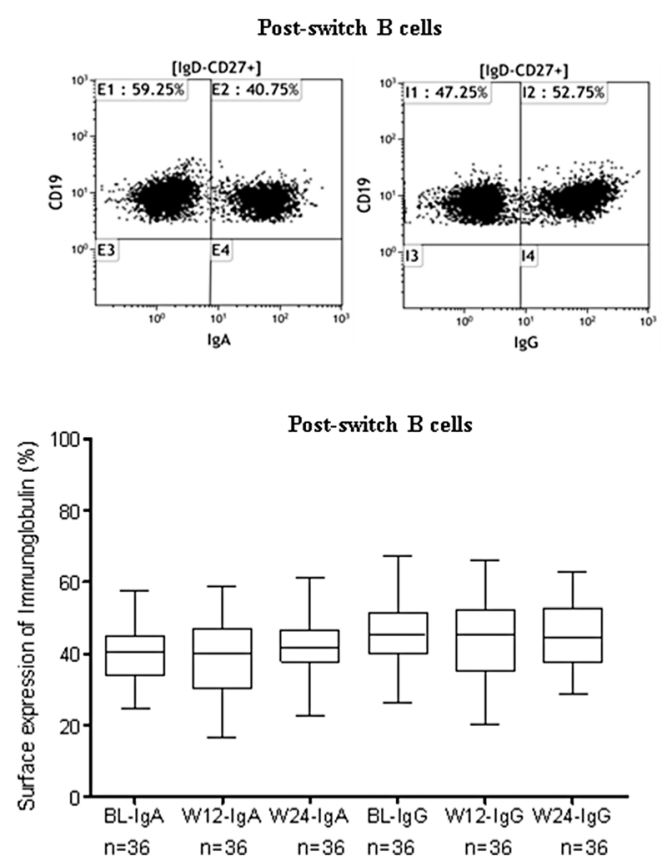

Figure 2 Surface expression of immunoglobulin isotypes during IL-6R inhibition. (A) Representative FACS plots showing the expression of immunoglobulin isotypes, IgA, IgG and IgM on gated DN and post-switch B cells. (B) During IL-6R inhibition, IgA+ DN B cells decreased significantly from 24.7 (10.0 to 64.2) percent median (range) to 18.4 (4.8 to 34.7) at week $12(P=0.004)$ and 20.5 (4.6 to 33.8) at week $24(P=0.04)$ respectively. There were no remarkable changes in relative $\lg \mathrm{G}+$ and $\operatorname{lgM}+\mathrm{DN} B$ Cells. (C) $\lg \mathrm{A}+$ and $\lg \mathrm{G}+$ post-switch B cells were not influenced during IL-6R inhibition. BL, baseline, W12, week 12 and W24, week 24. DN, double-negative; Ig, immunoglobulin; IL-6R, interleukin-6 receptor.

activity. During TCZ, DAS28 was reduced from a mean of 5.2 at baseline to 3.3 at week $12,2.7$ at week 24 and 1.9 at 1 year. During anti-TNF- $\alpha$ therapy DAS28 declined from a mean of 4.7 at BL to 3.1 at week $12,2.7$ at week 24 and 1.6 at 1 year. These results indicate that anti-TNF- $\alpha$ therapy does not influence the process of somatic hypermutation of $\mathrm{DN} B$ cells as is observed during IL-6R inhibition.

Ig-specific isotypes are modulated during IL-6R inhibition Since DN B cells are a heterogeneous population of IgG, IgA and IgM isotypes, we addressed if the TCZ therapy has distinct influences on different isotypes. We performed isotype-specific PCRs from single-sorted DN B cells, and amplified the $C_{H} 1 \mu-, C_{H} 1 \gamma$ - and $C_{H} 1 \alpha$-specific immunoglobulin gene rearrangements (Figure $3 \mathrm{C}$ ). At baseline, the isotype-specific Ig- $\mathrm{R}$ analysis revealed a significantly higher mutational frequency of IgA+ DN B cells $(P=0.01)$ compared to IgG $+\mathrm{DN} B$ cells at BL. As expected IgM+ DN B cells showed the lowest mutational frequency. However during TCZ therapy, all three isotypes $(\operatorname{IgA}+, \operatorname{IgG}+$ and $\operatorname{IgM}+)$ of $\mathrm{DN} B$ cells showed a significantly reduced mutational frequency. Mutational frequency of IgA + cells were found to be reduced (mean \pm standard error of the mean (SEM)) from $5.42 \pm 0.30 \%$ (BL) to $3.35 \pm 0.22 \%$ at week $12(P<0.0001), 2.41 \pm 0.34 \%$ at week $24(P<0.0001)$ and $2.26 \pm 0.33 \%(P<0.0001)$ at 1 year, respectively. The mutational frequency of IgG+ cells reduced from $4.45 \pm 0.20 \%$ (BL) to $2.51 \pm 0.22 \%$ at week 12 $(P<0.0001)$, to $2.16 \pm 0.23 \%$ at week $24(P<0.0001)$ and $2.00 \pm 0.20 \%(P<0.0001)$ at 1 year, respectively. Similarly, the mutational frequency of IgM+ cells also decreased from $2.87 \pm 0.32 \%$ (BL) to $1.55 \pm 0.23 \%$ at week $12(P=0.0016), 0.98 \pm 0.21 \%$ at week $24 \quad(P=0.0008)$ and $0.67 \pm 0.17 \%(P<0.0001)$ during 1 -year TCZ treatment (Figure 3C). These data are consistent with the overall $\mathrm{V}_{\mathrm{H}} 3$ analysis of individual $\mathrm{DN}$ B cells during TCZ treatment (Figure 3A).

\section{CDR3 length and targeting of mutational hotspots motifs during IL-6R inhibition}

Along with SHM, the length of the third complementary determining region (CDR3) is considered a signature of antigen contact and $\mathrm{T}$ cell help, and it represents imprints of selection. CDR3 lengths of $\mathrm{V}_{\mathrm{H}}$ gene rearrangements at different time points are shown in Figure 4A. We showed that CDR3 length of DN B cells increased from median (range) 45.0 (16.0 to 81.0) base pair (bp) (BL) to $48.0(18.0$ to 85.0$)$ bp at week $12(P=0.0004)$, 48.0 (18.0 to 85.0) bp at week $24(P<0.0001)$ and 50 (21.0 to 91.0$)$ bp $(P<0.0001)$ at 1 year during TCZ therapy (Figure 4A). Analysis of the frequency of targeted RGYW/WRCY mutations ( $\mathrm{R}$, purine; Y, pyrimidine; W, $\mathrm{A} / \mathrm{T}$ ) showed that it decreased significantly from median 


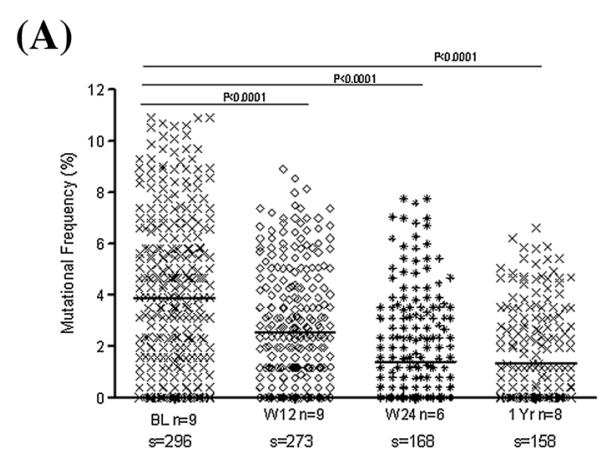

(B)

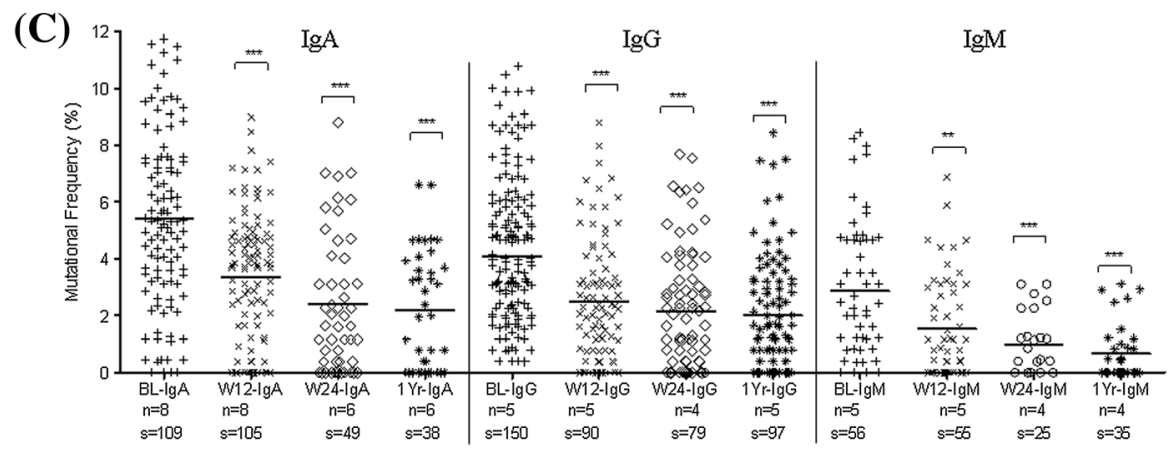

Figure 3 Ig-receptor somatic hypermutation of gene rearrangements during IL-6R and TNF- $\alpha$ inhibition in DN B cells. (A) lg- $V_{H} 3$ gene rearrangements during $\mathrm{IL}-6 \mathrm{R}$ inhibition. Reduction in mutational frequency of $\mathrm{Ig}-\mathrm{V}_{\mathrm{H}} 3$ gene rearrangements of DN $B$ cells from the peripheral blood of RA patients during TCZ therapy. The mutational frequency was significantly reduced at week 12,24 and 1 year ( ${ }^{* * *} P<0.0001$ compared to BL). (B) Ig-receptor somatic hypermutation of $\mathrm{V}_{\mathrm{H}} 3$ gene rearrangements during TNF-a inhibition. During TNF-a inhibition by adalimumab, a comparable mutational frequency of $V_{H}$ gene rearrangements of DN B cells from the peripheral blood of RA patients were observed at all time points. (C) Ig-receptor somatic hypermutation of isotype-specific $\lg A+\lg \mathrm{G}+$ and $\lg \mathrm{M}+$ gene rearrangements during IL-6R inhibition in DN B cells. At the $B L$, the mutational frequency of IgA+ DN B cells is significantly higher compared to IgG+ and IgM+ DN B cells. During TCZ therapy, all isotypes $\lg A+, \lg G+$ and IgM+ DN B cells showed a significantly reduced mutational frequency $\left({ }^{* * *} P<0.0001{ }^{* * *} P<0.001\right)$. In a scatter plot, the line represents mean of all values and each dot depicts the mutational frequency of a single sequence. $P$ values were determined by Wilcoxon test using GraphPad Prism 5. (BL, baseline; W12, week 12; W24, week 24; n, number of individuals; s, number of sequence analyzed). DN, double-negative; HD, healthy donor; Ig, immunoglobulin; IL-6R, interleukin-6 receptor; RA, rheumatoid arthritis; TCZ, tocilizumab; TNF-a, tumor necrosis factor alpha.

(range) 24.6 (21.1 to 38.9$)$ at baseline to 20.5 (17.7 to 26.1) at week $12(P=0.046), 20.7(14.9$ to 23.4$)$ at week 24 $(P=0.004)$ and $19.2(12.6$ to 24.5$)$ at 1 year $(P=0.004)$ during TCZ therapy (Figure 5A). Frequency analysis of mutational hotspot targeting and CDR3 length analysis during anti-TNF- $\alpha$ therapy also revealed no changes in DN B cells Ig-receptor (Figures 4B and 5B). Furthermore, the frequency analysis of targeted mutations revealed that there was a significant decrease of this targeted mutations from baseline to week 12, 24 and 1 year in all isotypespecific IgA+, IgG+ and IgM+ DN B cells during TCZ therapy (Figure 5C). Likewise, CDR3 length of isotypespecific DN B cells increased from BL to week 12, week 24 and 1 year during TCZ therapy (Figure 4C). Overall, these findings indicate that TCZ therapy not only modulates acquired SHMs in Ig-R of DN B cells, but also exerts effects on the length of CDR3.

\section{Discussion}

With increased understanding of mechanistic pathways of B cell involvement in autoimmune diseases, a number of studies have provided evidence that $B$ cells play a central role in the pathogenesis of RA and that certain subsets may be exploitable as biomarkers. Memory B cells, in particular, seem to be important in driving chronic inflammation [28,34]. Targeting pre-switch and post-switch memory B cells has been found to be important for the response to rituximab [35]. However the impact of different memory B cell subsets has not been thoroughly studied. We recently showed that in vivo IL$6 \mathrm{R}$ inhibition by TCZ does influence the peripheral preswitch and post-switch memory B cells in RA [14,25]. Because CD27-IgD- DN B cells also carry features of memory B cells $[19,20]$, we were particularly interested in studying their behavior in active RA, since there is scant literature available for RA. DN B cells confer a notable component of the peripheral blood B cell compartment in RA patients (Figure 1A). Similar to others $[35,36]$, we found a relatively higher frequency of the DN B cell subset in RA patients compared to healthy donors, whereas the absolute numbers of DN B cells were comparable to $\mathrm{HD}$ is mainly due to lymphopenia 
(A)

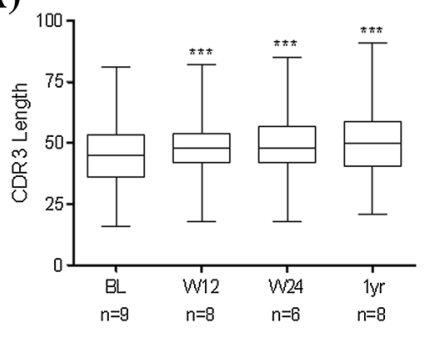

(B)

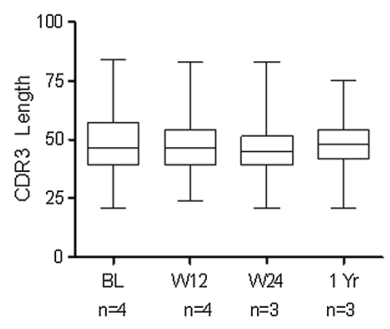

(C)

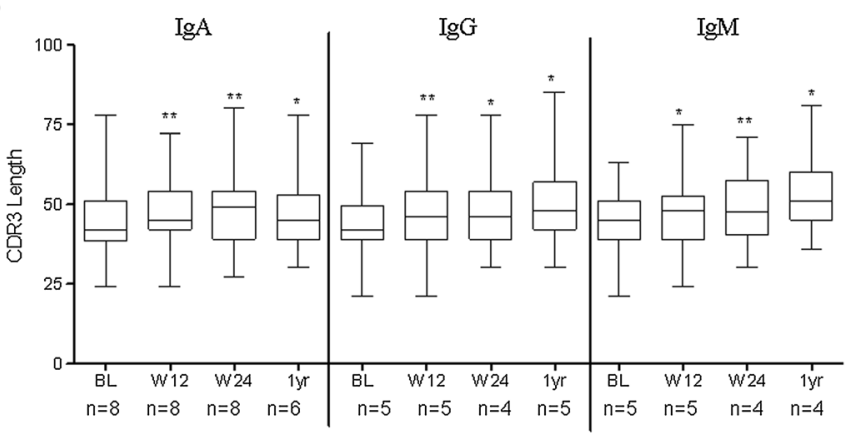

Figure 4 CDR3 length during IL-6R and TNF-a inhibition. (A) Significant increase in the CDR3 length of Ig- $V_{H}$ gene rearrangements of DN $B$ cells from the peripheral blood of RA patients during IL-6R inhibition. ( $\left.{ }^{* * *} P<0.0001\right)$. (B) During TNF-a inhibition, a comparable CDR3 length of Ig- $V_{H}$ gene rearrangements of DN B cells were observed at all time points. (C) There is significant increase in the length of the CDR3 in all three isotypes specific to DN B cells during IL-6R inhibition. P values were determined by Wilcoxon test using GraphPad Prism 5. CDR3, the length of the third complementary determining region; DN, double negative; IL-6R, interleukin-6 receptor; RA, rheumatoid arthritis; TNF-a, tumor necrosis factor alpha.

(A)

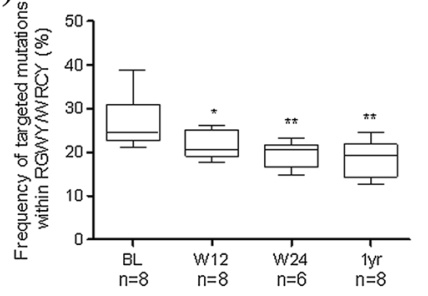

(C)

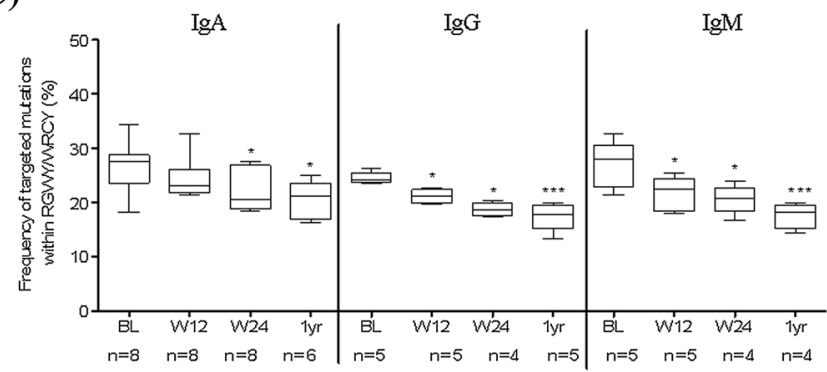

(B)

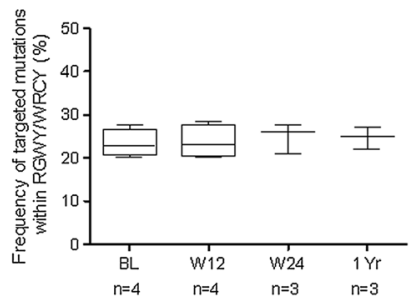

$\operatorname{IgM}$

Figure 5 Frequency of targeted mutations within hotspot motifs during IL-6R and TNF-a inhibition. (A) A significant reduction in frequency of targeted mutations within RGWY WRCY is found in Ig- $V_{H}$ gene rearrangements of DN B cells during TCZ therapy $\left({ }^{*} P=0.046,{ }^{* *} P=0.004\right)$. (B) During TNF-a inhibition, a comparable mutational hotspot targeting of $V_{H}$ gene rearrangements of DN B cells were observed at all time points. (C) $A$ significant reduction in frequency of targeted mutations within RGWY/WRCY is found in all three isotypes specific to DN B cells during IL-6R inhibition. $P$ values were determined by Wilcoxon test using GraphPad Prism 5. DN, double negative; Ig, immunoglobulin; IL-6R, interleukin-6 receptor; TCZ, tocilizumab; TNF-a, tumor necrosis factor alpha. 
in RA patients. Elevated DN memory B cells have been described to be related to aging, persistent antigen stimulation and seem to reflect overall B-cell hyperactivity [19,23,37-39]. In our study, elevation of DN B cells did not reflect simply inflammation since their number did not correlate to CRP or DAS28 levels.

We describe a heterogeneous DN B cell population containing IgG+, IgA+ and IgM+ DN B cells with predominance of the IgG clearly different from post-switch memory B cells (Figure 2A). During TCZ therapy, there was moderate influence on the phenotypic composition of the DN compartment. Nevertheless, the IgA-DN B cell phenotype seems to be particularly amenable to IL$6 \mathrm{R}$ inhibition - the percentage of IgA+ DN B cells significantly decreased during TCZ therapy (Figure 2B). Also absolute numbers of IgA+ DN cells were reduced significantly (Additional file 3 ). Moreover we see a significant reduction of serum IgA (Table 1 and reference [14]) as well as RF-IgA levels (Table 1). This highlights the IL-6 susceptibility of the IgA isotype. However, it should be pointed out that our data provides no direct relation of IgA-DN B cells to declining IgA serum factors. Nevertheless IL-6 has been ascribed a critical player in the development of local $\operatorname{IgA}$ antibody responses and a remarkable reduction of mucosal IgA-producing cells has been reported in mice with targeted disruption of the gene that encodes IL-6 [40]. Future experiments need to address how peripheral IgA + DN B cells may be related to the mucosal immune system.

SHM of immunoglobulin gene rearrangements is a hallmark of B cell maturation into memory cells after antigen encounter. Despite their mainly switched phenotype, we also found the mutational frequency of DN B cells in RA to be significantly lower compared to post-switch B cells and similar to pre-switch B cells. This has been previously reported for other diseases and older people [19,20,24,25]. We found a significant decrease in mutational frequency of the B cell receptor (BCR) at week 12, 24 and over a year during TCZ therapy (Figure 3A). TCZ treatment again induces profound changes with reduced mutational status in all three isotypes (Figure 3C). Interestingly, IgA+ DN B cells also harbored the highest mutational frequency compared to IgG+ and IgM+ DN B cells, which was evident in RA as well as healthy donors (Additional file 2B).

It is known that SHM favors defined mutational hotspots. In particular, RGYW/WRCY motif mutations are preferentially targeted by $\mathrm{T}$ cell-dependent signals including CD40-CD40 ligand interactions [41]. The decrease in frequency of targeted mutations within RGYW/WRCY and increase in CDR3 during IL-6R inhibition indicates that during therapy the DN B cell population consists of less antigen-experienced $B$ cells. This effect seems mainly influenced by IL-6R inhibition therapy since we did not observe any change in these parameters during anti-TNF- $\alpha$ therapy using adalimumab (Figure 3B), although both biologics resulted in good reduction of clinical inflammation. In a previous study looking at the pre-switch B cell compartment, we observed a similar pattern with adalimumab failing to influence Ig-R mutation [25]. Therefore, it seems likely that IL-6R blockade does affect B cell maturation in vivo to a substantial extent. However, at the moment it is not clear how direct and indirect effects on $\mathrm{B}$ cells contribute to the observed effects since TCZ is also reported to reduce activated CD4+ T cells as early as 12 weeks after treatment [42]. Data still leaves these questions open. DN B cells are hypothesized to resemble transient effector B cells [43]. A recent report suggested that Syk++ B cells lacking CD27 expression represent a unique atypical memory-like $B$ cell, which may be relevant for IgG+ plasmablast generation [44]. Furthermore they might derive from incomplete GC or alternatively from extrafollicular reactions [43]. Since mutational frequencies of DN and pre-switch B cells are significantly lower than typical post-switch B cells, it might be suggested that either of these cells are pregerminal centre or leave the GCs quickly by shedding CD27. On the other hand, DN B cells still could fail to upregulate CD27 expression before leaving GCs since CD27-negative memory B cells have also been found and isolated from human tonsils [45]. Since shedding or downregulation of CD27 have been reported as an indication of continuous antigen stimulation and $\mathrm{T}$ cell exhaustion, our observed connection of DN B cells numbers to response to IL-6R inhibition may reflect a state of chronic B cell hyperactivity closely linked to IL-6 [37-39]. Furthermore, the data are consistent with the idea that DN memory B cells are distinctly generated or have a shorter half-life than conventional post-switch memory $B$ cells resulting in a measurable impact of IL-6R blockade on DN B cells.

The search for predictive biomarkers to individualize treatment strategies in RA is of current interest. For IL6R inhibition with TCZ, predictive markers for response have not yet been identified. In our study, we were able to relate the level of peripheral DN B cells to the clinical response to TCZ (Figure 1B-C). Patients achieving EULAR good response at week 12 had significantly lower percentages and absolute numbers of DN B cells at baseline before TCZ therapy. In univariate logistic regression analysis, a lower frequency of DN B cells showed an odds ratio of 1.48 for achieving a EULAR good response to TCZ. It is important to note that the frequency of DN B cells is not correlated to CRP or DAS28 levels at baseline. Thus CD27-IgD- DN B cells may indicate an elevated B cell activity in RA that is not reflected by these inflammatory markers but is susceptible to TCZ. This may allow future studies to exploit DN B cells as biomarkers for response to TCZ. 


\section{Conclusions}

In summary, we demonstrate a significantly higher population of CD27-IgD- DN B cells in RA patients. These DN $B$ cells are a mixture of somatically mutated IgG, IgA and IgM isotype-bearing cells with a dominance of IgG isotype. TCZ therapy results in a decrease in the frequency of IgA+ DN B cells in particular. In parallel, a decrease of serum IgA and RF-IgA levels is observed. On the molecular level, TCZ therapy reduces the mutational frequency and RGYW hotspot targeting in all DN B cells over a period of 1 year. Based on these current findings, DN memory B cells may serve as a candidate biomarker for response to TCZ therapy as lower baseline values of these cells were related to higher proportions of EULAR good responders.

\section{Additional files}

Additional file 1: Surface expression of DN B cells immunoglobulin isotypes in HD and RA patients. (A) The distribution of $\lg A+\lg G+$ and IgM+DN B cells were comparable in HD and RA patients before undergoing therapy. (B) Similarly in post-switch memory B cells, distributions of $\lg A+$ and $\lg G+$ cells were comparable.

Additional file 2: Ig-receptor somatic hypermutation of $\mathrm{V}_{\mathrm{H}} 3$ and isotype-specific $\lg A+, \lg G+$ and $\lg M+$ gene rearrangements of $D N B$ cells in HD and RA. (A) Comparable mutational frequency of $\mathrm{V}_{\mathrm{H}} 3$ gene rearrangements of DN B cells from the peripheral blood of RA patients before undergoing therapy and HD. (B) Isotype specific IgA+, IgG+ and lgM+ gene rearrangements of DN B cells were comparable.

Additional file 3: Surface expression of immunoglobulin isotypes during IL-6R inhibition (absolute cell numbers). (A) IgA+ DN B cell absolute numbers per $\mu \mathrm{l}$ are decreased significantly from median (range) 1.9 (0.42 to 9.5$)$ to $1.4(0.4$ to 6.0$)$ at week $24(P=0.04)$. Absolute cell numbers of $\mathrm{lgG}+$ and $\operatorname{lgM}+\mathrm{DN} B$ cells are showing a trend for reduced numbers during TCZ therapy. (B) Absolute cell numbers of isotype specific lgG+ and IgA+ post-switch B cells shows a weak trend to reduced numbers during TCZ therapy. Total number of patients $=36$. $\mathrm{BL}=$ baseline, $\mathrm{W} 12=$ week 12 and $\mathrm{W} 24=$ week $24 . P$ values were determined by Mann-Whitney $U$ test using GraphPad Prism 5. $\left({ }^{* * *} P<0.0001\right.$, ${ }^{* *} P<0.001$ and $\left.{ }^{*} P<0.05\right)$.

\section{Abbreviations}

ABCB1: ATP-binding cassette B1 transporter; ACPA-positive: anti-citrullinated protein antibodies; ADA: adalimumab; BL: baseline; bp: base pair; BSA: bovine serum albumin; BCR: B cell receptor; CDR3: length of the third complementary determining region; CRP: C-reactive protein; DAS28: disease activity score using 28 joint counts; DMARDs: disease-modifying antirheumatic drugs; DN: double negative; ESR: erythrocyte sedimentation rate; EULAR: The European League Against Rheumatism; GC: germinal center; HD: healthy donor; Ig-R: immunoglobulin receptor; IgD: immunoglobulin D; IL-6R: interleukin-6 receptor; mAb: monoclonal antibody; MTX: methotrexate; PBMCs: peripheral blood mononuclear cells; PCR: polymerase chain reaction; RA: rheumatoid arthritis; RF: rheumatoid factor; SHM: somatic hypermutation; SLE: systemic lupus erythematosus; TCZ: tocilizumab; Th: T helper; TNF-a: tumor necrosis factor alpha; TNFR: tumor necrosis factor receptor.

\section{Competing interests}

The authors declare that they have no competing interests.

\section{Authors' contributions}

ZM carried out the molecular and flow cytometer data acquisition and their analyses, performed the statistics for molecular and clinical data, and drafted the manuscript. KM performed the molecular experiments and was involved in revising the manuscript critically for important intellectual content. PR and MS were involved in clinical studies and revising the manuscript. TD provided reagents and critically revised the manuscript. ZM, KM and H-PT designed the project and wrote the manuscript. All authors approved the final version to be published. H-PT and ZM had full access to all of the data in the study and take responsibility for the integrity of the data and the accuracy of the data analysis.

\section{Acknowledgements}

The authors thank Katharina Eckert for her technical assistance in molecular work and also Anette Koss-Kinzinger, Christian Linden and Yvonne Gold for their technical assistance in flow cytometer work. The authors thank Dr. Swati Singh for checking grammatical errors in the manuscript. This work was funded by Interdisziplinäres Zentrum für Klinische Forschung (IZKF) Würzburg project A-201 and the publication was funded by the German Research Foundation (DFG) and the University of Wuerzburg in the funding programme Open Access Publishing.

\section{Author details}

${ }^{1}$ Department of Medicine II, Rheumatology and Clinical Immunology, University of Würzburg, Oberdürrbacher Str. 6, D-97080 Würzburg, Germany. ${ }^{2}$ Department of Molecular Pathology, Institute of Pathology University of Würzburg, Josef-Schneider-Str. 2, D-97080 Würzburg, Germany. ${ }^{3} \mathrm{CC} 12$, Department of Medicine /Rheumatology and Clinical Immunology, Charite Universitätsmedizin Berlin and DRFZ Berlin, Charitéplatz 1, D-10117 Berlin, Germany.

Received: 13 October 2014 Accepted: 23 February 2015

Published online: 14 March 2015

\section{References}

1. Klareskog L, Catrina Al, Paget S. Rheumatoid arthritis. Lancet. 2009;373:659-72.

2. Roll P, Mahmood Z, Muhammad K, Feuchtenberger M, Dörner T, Tony HP. Long term repopulation of peripheral $B$ cell subsets after single and repeated rituximab infusions in patients with rheumatoid arthritis. Clin Exp Rheumatol. 2015. In press (article code: CER7847)

3. Martin F, Chan AC. B cell immunobiology in disease: evolving concepts from the clinic. Annu Rev Immunol. 2006:24:467-96.

4. Fillatreau S. Cytokine-producing B, cells as regulators of pathogenic and protective immune responses. Ann Rheum Dis. 2013; 72 Suppl 2:ii80-4.

5. Mclnnes IB, Schett G. Cytokines in the pathogenesis of rheumatoid arthritis. Nat Rev Immunol. 2007;7:429-42.

6. Voulgari PV, Drosos AA. Adalimumab in the treatment of rheumatoid arthritis. Expert Opin Biol Ther. 2014. [Epub ahead of print].

7. Carbone G, Wilson A, Diehl SA, Bunn J, Cooper SM, Rincon M. Interleukin-6 receptor blockade selectively reduces $\mathrm{IL}-21$ production by CD4 T cells and IgG4 autoantibodies in rheumatoid arthritis. Int J Biol Sci. 2013;9:279-88.

8. Mihara M, Hashizume M, Yoshida H, Suzuki M, Shiina M. IL-6/IL-6 receptor system and its role in physiological and pathological conditions. Clin Sci. 2012;122:143-59.

9. Dorner T, Lipsky PE. B cells: depletion or functional modulation in rheumatic diseases. Curr Opin Rheumatol. 2014;26:228-36.

10. Tanaka T, Narazaki M, Kishimoto T. Therapeutic targeting of the interleukin- 6 receptor. Annu Rev Pharmacol Toxicol. 2012;52:199-219.

11. Garnero P, Thompson E, Woodworth T, Smolen JS. Rapid and sustained improvement in bone and cartilage turnover markers with the anti-interleukin- 6 receptor inhibitor tocilizumab plus methotrexate in rheumatoid arthritis patients with an inadequate response to methotrexate: results from a substudy of the multicenter double-blind, placebo-controlled trial of tocilizumab in inadequate responders to methotrexate alone. Arthritis Rheum. 2010;62:33-43.

12. Nishimoto N, Terao K, Mima T, Nakahara H, Takagi N, Kakehi T. Mechanisms and pathologic significances in increase in serum interleukin-6 (IL-6) and soluble IL-6 receptor after administration of an anti-IL-6 receptor antibody, tocilizumab, in patients with rheumatoid arthritis and Castleman disease. Blood. 2008;112:3959-64.

13. Souto-Carneiro MM, Mahadevan V, Takada K, Fritsch-Stork R, Nanki T, Brown $M$, et al. Alterations in peripheral blood memory B cells in patients with active rheumatoid arthritis are dependent on the action of tumour necrosis factor. Arthritis Res Ther. 2009;11:R84.

14. Roll P, Muhammad K, Schumann M, Kleinert S, Einsele H, Dorner T, et al. In vivo effects of the anti-interleukin- 6 receptor inhibitor tocilizumab on the $B$ cell compartment. Arthritis Rheum. 2011;63:1255-64. 
15. Goodwin RG, Alderson MR, Smith CA, Armitage RJ, VandenBos T, Jerzy R, et al. Molecular and biological characterization of a ligand for CD27 defines a new family of cytokines with homology to tumor necrosis factor. Cell. 1993:73:447-56.

16. Klein U, Goossens T, Fischer M, Kanzler H, Braeuninger A, Rajewsky K, et al. Somatic hypermutation in normal and transformed human B cells. Immunol Rev. 1998;162:261-80.

17. Wang J, Shan Y, Jiang Z, Feng J, Li C, Ma L, et al. High frequencies of activated $B$ cells and follicular helper $T$ cells are correlated with disease activity in patients with new onset rheumatoid arthritis. Clin Exp Immunol. 2013;174:212-20.

18. Shi Y, Agematsu K, Ochs HD, Sugane K. Functional analysis of human memory B-cell subpopulations: IgD + CD27+ B cells are crucial in secondary immune response by producing high affinity lgM. Clin Immunol. 2003;108:128-37

19. Wei C, Anolik J, Cappione A, Zheng B, Pugh-Bernard A, Brooks J, et al. A new population of cells lacking expression of CD27 represents a notable component of the B cell memory compartment in systemic lupus erythematosus. J Immunol. 2007:178:6624-33.

20. Fecteau JF, Cote G, Neron S. A new memory CD27-lgG+ B cell population in peripheral blood expressing $\mathrm{VH}$ genes with low frequency of somatic mutation. J Immunol. 2006;177:3728-36.

21. Jacobi AM, Reiter K, Mackay M, Aranow C, Hiepe F, Radbruch A, et al. Activated memory B cell subsets correlate with disease activity in systemic lupus erythematosus: delineation by expression of CD27, IgD, and CD95. Arthritis Rheum. 2008;58:1762-73.

22. Wirths $\mathrm{S}$, Lanzavecchia $\mathrm{A}$. ABCB1 transporter discriminates human resting naive $B$ cells from cycling transitional and memory B cells. Eur J Immunol. 2005;35:3433-41.

23. Colonna-Romano G, Bulati M, Aquino A, Pellicanò $M$, Vitello S, Lio D, et al. A double-negative (lgD - CD27-) B cell population is increased in the peripheral blood of elderly people. Mech Ageing Dev. 2009;130:681-90.

24. Buffa S, Bulati M, Pellicano M, Dunn-Walters DK, Wu YC, Candore G, et al. B cell immunosenescence: different features of naive and memory $B$ cells in elderly. Biogerontology. 2011;12:473-83.

25. Muhammad K, Roll P, Seibold T, Kleinert S, Einsele H, Dorner T, et al. Impact of IL-6 receptor inhibition on human memory B cells in vivo: impaired somatic hypermutation in preswitch memory B cells and modulation of mutational targeting in memory B cells. Ann Rheum Dis. 2011;70:1507-10.

26. Aletaha D, Neogi T, Silman AJ, Funovits J, Felson DT, Bingham 3rd CO, et al. 2010 Rheumatoid arthritis classification criteria: an American College of Rheumatology/European League Against Rheumatism collaborative initiative. Arthritis Rheum. 2010;62:2569-81.

27. van Riel PL. The development of the disease activity score (DAS) and the disease activity score using 28 joint counts (DAS28). Clin Exp Rheumatol. 2014; 32:S-65-74.

28. Muhammad $K$, Roll $P$, Einsele $H$, Dorner $T$, Tony HP. Delayed acquisition of somatic hypermutations in repopulated IGD + CD27+ memory B cell receptors after rituximab treatment. Arthritis Rheum. 2009:60:2284-93.

29. Ruzickova S, Pruss A, Odendahl M, Wolbart K, Burmester GR, Scholze J, et al. Chronic lymphocytic leukemia preceded by cold agglutinin disease: intraclonal immunoglobulin light-chain diversity in $\mathrm{V}(\mathrm{H}) 4-34$ expressing single leukemic B cells. Blood. 2002;100:3419-22.

30. Hansen A, Gosemann M, Pruss A, Reiter K, Ruzickova S, Lipsky PE, et al. Abnormalities in peripheral B cell memory of patients with primary Sjogren's syndrome. Arthritis Rheum. 2004;50:1897-908.

31. Dorner T, Brezinschek HP, Brezinschek RI, Foster SJ, Domiati-Saad R, Lipsky $P E$. Analysis of the frequency and pattern of somatic mutations within nonproductively rearranged human variable heavy chain genes. J Immunol. 1997:158:2779-89.

32. Souto-Carneiro MM, Longo NS, Russ DE, Sun HW, Lipsky PE. Characterization of the human lg heavy chain antigen binding complementarity determining region 3 using a newly developed software algorithm, JOINSOLVER. J Immunol. 2004;172:6790-802.

33. Kaminski DA, Wei C, Qian Y, Rosenberg AF, Sanz I. Advances in human B cell phenotypic profiling. Front Immunol. 2012;3:302.

34. Edwards JC, Szczepanski L, Szechinski J, Filipowicz-Sosnowska A, Emery P, Close DR, et al. Efficacy of B-cell-targeted therapy with rituximab in patients with rheumatoid arthritis. N Engl J Med. 2004;350:2572-81.

35. Brezinschek HP, Rainer F, Brickmann K, Graninger WB. B lymphocyte-typing for prediction of clinical response to rituximab. Arthritis Res Ther. 2012;14:R161.
36. de la Torre I, Moura RA, Leandro MJ, Edwards J, Cambridge G. B-cell-activating factor receptor expression on naive and memory B cells: relationship with relapse in patients with rheumatoid arthritis following B-cell depletion therapy. Ann Rheum Dis. 2010:69:2181-8.

37. Swaak AJ, Hintzen RQ, Huysen V, van den Brink HG, Smeenk JT. Serum levels of soluble forms of T cell activation antigens CD27 and CD25 in systemic lupus erythematosus in relation with lymphocytes count and disease course. Clin Rheumatol. 1995;14:293-300.

38. De Jong R, Brouwer M, Hooibrink B, Van der Pouw-Kraan T, Miedema F, Van Lier RA. The CD27- subset of peripheral blood memory CD4+ lymphocytes contains functionally differentiated T lymphocytes that develop by persistent antigenic stimulation in vivo. Eur J Immunol. 1992;22:993-9.

39. Kai K, Rikiishi H, Sugawara S, Takahashi M, Takada H, Kumagai K. Lipopolysaccharide-dependent down-regulation of CD27 expression on T cells activated with superantigen. Immunology. 1999:98:289-95.

40. Ramsay AJ, Husband AJ, Ramshaw IA, Bao S, Matthaei KI, Koehler G, et al. The role of interleukin-6 in mucosal IgA antibody responses in vivo. Science. 1994;264:561-3.

41. Monson NL, Foster SJ, Brezinschek HP, Brezinschek RI, Dorner T, Lipsky PE. The role of CD40-CD40 ligand (CD154) interactions in immunoglobulin light chain repertoire generation and somatic mutation. Clin Immunol. 2001;100:71-81.

42. Shirota Y, Yarboro C, Fischer R, Pham TH, Lipsky P, Illei GG. Impact of anti-interleukin- 6 receptor blockade on circulating $T$ and $B$ cell subsets in patients with systemic lupus erythematosus. Ann Rheum Dis. 2013;72:118-28.

43. Sanz I, Wei C, Lee FE, Anolik J. Phenotypic and functional heterogeneity of human memory B cells. Semin Immunol. 2008;20:67-82

44. Fleischer SJ, Giesecke C, Mei HE, Lipsky PE, Daridon C, Dorner T. Increased frequency of a unique spleen tyrosine kinase (Syk) bright memory B-cell population in systemic lupus erythematosus. Arthritis Rheum. 2014;66:3424-35.

45. Ehrhardt GR, Hsu JT, Gartland L, Leu CM, Zhang S, Davis RS, et al. Expression of the immunoregulatory molecule FCRH4 defines a distinctive tissue-based population of memory B cells. J Exp Med. 2005;202:783-91.

\section{Submit your next manuscript to BioMed Central and take full advantage of:}

- Convenient online submission

- Thorough peer review

- No space constraints or color figure charges

- Immediate publication on acceptance

- Inclusion in PubMed, CAS, Scopus and Google Scholar

- Research which is freely available for redistribution 